\title{
Precision and resolution in laser direct microstructuring with bursts of picosecond pulses
}

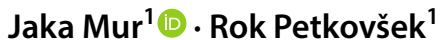

Received: 26 September 2017 / Accepted: 16 December 2017 / Published online: 23 December 2017

(c) Springer-Verlag GmbH Germany, part of Springer Nature 2017

\begin{abstract}
Pulsed laser sources facilitate various applications, including efficient material removal in different scientific and industrial applications. Commercially available laser systems in the field typically use a focused laser beam of 10-20 $\mu \mathrm{m}$ in diameter. In line with the ongoing trends of miniaturization, we have developed a picosecond fiber laser-based system combining fast beam deflection and tight focusing for material processing and optical applications. We have predicted and verified the system's precision, resolution, and minimum achievable feature size for material processing applications. The analysis of the laser's performance requirements for the specific applications of high-precision laser processing is an important aspect for further development of the technique. We have predicted and experimentally verified that maximal edge roughness of single-micrometer-sized features was below $200 \mathrm{~nm}$, including the laser's energy and positioning stability, beam deflection, the effect of spot spacing, and efficient isolation of mechanical vibrations. We have demonstrated that a novel fiber laser operating regime in bursts of pulses increases the laser energy stability. The results of our research improve the potential of fiber laser sources for material processing applications and facilitate their use through enabling the operation at lower pulse energies in bursts as opposed to single pulse regimes.
\end{abstract}

\section{Introduction}

Pulsed laser sources have been used for various material processing applications in both scientific and industrial environments. Historically, the use and comparison of ultrashort pulse laser sources began with investigation of ablation of solids [1]. The field has evolved through numerous fundamental studies, for example [2-9]. The advantages of using picosecond or shorter laser pulses have been recognized and evaluated in said studies regarding the localized energy deposition, reduced heat effects, precise structure definition, ability to structure transparent materials through multiphoton processes, and low ablation thresholds. Contemporary ultra-short pulse lasers are also used in industrial applications due to an achievable high structure quality and throughput $[10,11]$. In recent studies, desirable effects of

Jaka Mur

jaka.mur@fs.uni-lj.si

Rok Petkovšek

rok.petkovsek@fs.uni-lj.si

1 Faculty of Mechanical Engineering, University of Ljubljana, Aškerčeva 6, 1000 Ljubljana, Slovenia novel laser operation regimes have been recognized, such as the use of double pulses [11, 12] or bursts of pulses [13-18].

Commercially available laser systems in the field of material processing typically use a focused laser beam of $10-20 \mu \mathrm{m}$ in diameter. In line with the ongoing trends of miniaturization in industry and emerging scientific disciplines such as nanophotonics, micro- and nanofluidics, and nanoelectronics, already several laser direct structuring devices for sub-micrometer lithography [19] and selective laser-induced wet etching of glass [20] have been developed. Our aim has been to design and evaluate an experimental laser direct microstructuring device for material processing applications, capable of manufacturing single-micrometersized structures or smaller in an accessible and chemical-free prototyping platform.

We have used our own picosecond fiber laser source to achieve the desired laser output. Fiber lasers are currently known for high average powers and exceptional beam quality [21], but their design becomes difficult and expensive if a high peak power, which may be reached with energetic picosecond or shorter laser pulses, is necessary. The combination of tight laser beam focusing, needed to achieve singlemicrometer features, and operation in bursts in pulses both circumvent this limitation and facilitate the use of a fiber 
laser. Tight focusing results in the required laser fluences at relatively low pulse energies, while operation in bursts of pulses contributes as well as long as the effect of a burst of pulses is at least comparable to a single pulse of equal total energy. The latter has been proven to hold true by other groups $[13,15]$ and our previous research [16].

We have identified the sources of beam instabilities on the sample that cannot be omitted by the device setup. While mechanical vibrations can be isolated using an optical table, the laser beam positioning and pulse energy stability, the beam steering mechanism, and geometrical effects of spot spacing must be sufficiently low by design. We have measured the laser properties and have predicted the resulting structure edge roughness according to the beam shape and size used, as well as the optical setup between the laser source and the sample. We have analyzed the laser's performance requirements for the specific application of highprecision laser processing. We have experimentally verified the device precision, repeatability, and isolation of mechanical vibrations, and compared the predictions with properties of actual structures in a thin indium tin oxide (ITO) layer. The results show that our fiber laser setup fulfills the requirement to achieve the needed degree of precision for optical applications and thin-layer material processing with a singlemicrometer feature size.

\section{Experimental device}

We have designed and evaluated an experimental direct laser microstructuring device, consisting of our own picosecond fiber-amplifier-based pulsed laser source [22], beam guiding optics including acousto-optic deflectors (AODs), automated sample movement table, CAD software, and control electronics. The setup was designed for laser processing with nanometer resolution and singlemicrometer feature size. The requirements were fulfilled using custom driver electronics and AODs for beam deflection (DTSXY400, AA Opto-Electronic) to circumvent the limitations of laser beam positioning with either scanner-based or table-based systems (limit on precision due to induced vibrations and mechanical acceleration).

The maximal repositioning frequency was defined by the active aperture of AODs and was up to $100 \mathrm{kHz}$. This also limits the highest repetition frequency of the laser if operation with single pulses is chosen. In the experiment, the AODs were synchronized to the fiber laser using external triggering at a working repositioning frequency of approximately $83.3 \mathrm{kHz}$ (labelled $f_{\mathrm{b}}$ in Fig. 1). The beam deflection efficiency was around $50 \%$ across the field after applying an equalization algorithm to compensate for the AODs native angular variations of deflection efficiency. The laser source was working at a $532 \mathrm{~nm}$ wavelength and was able to output tunable bursts of 65 ps pulses. Each time a deflection angle was set on the AODs, a single burst of 1, 5, 10, or 20 laser pulses (or PPB-pulses per burst) was transmitted to the defined spot on the material. The intra-burst repetition rate used was $40 \mathrm{MHz}$, labelled $f_{\mathrm{i}}$ in Fig. 1. Operation in bursts of pulses is thus defined as a regime where a quick succession of pulses repeats with a lower repetition frequency $f_{\mathrm{b}}$. Each burst was appropriately compensated to achieve approximately equal pulse energies throughout the burst.

We have focused the laser beam using a $20 \times$ magnification microscope objective (Nikon Plan Fluor $20 \times / 0.5$ ). The calculated focused beam diameter was below $1 \mu \mathrm{m}$, with a corresponding Rayleigh range of approximately $2 \mu \mathrm{m}$. Using the AODs have given us a working scan field size of $300 \times 300 \mu \mathrm{m}^{2}$. With a combination of sample movement and compensation of the detected final table position, we were able to precisely combine multiple scan fields over centimeter distances. A schematic illustration of the experimental device setup is shown in Fig. 1, including a representation of stitched scan fields on the material.

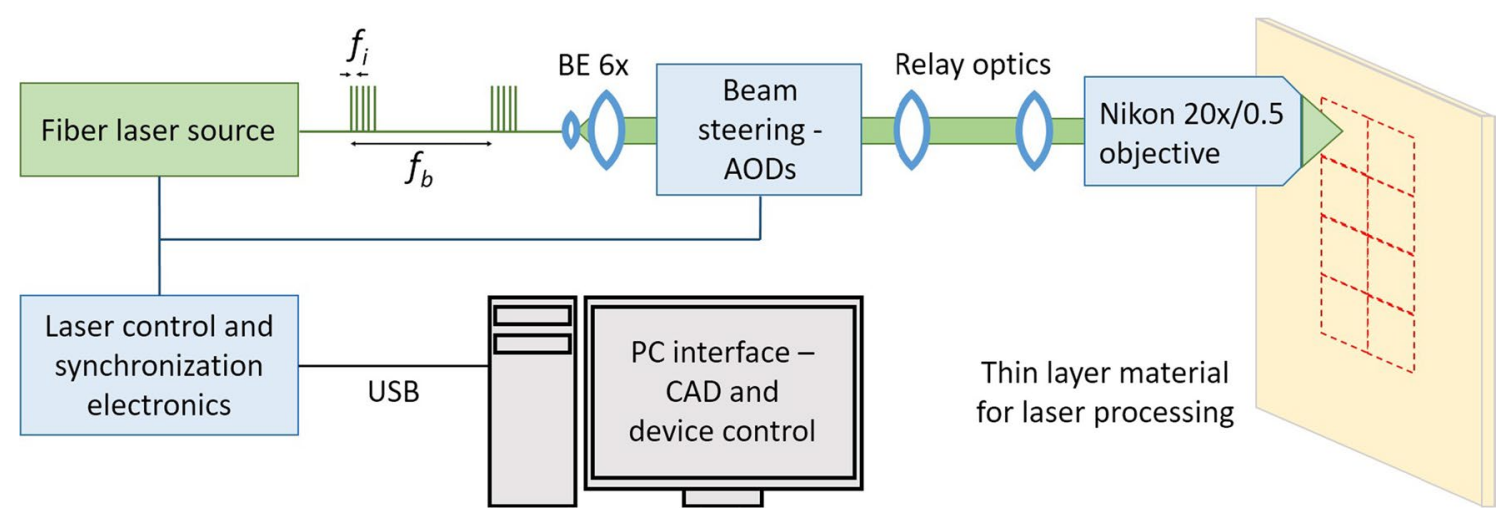

Fig. 1 Schematic representation of the experimental device setup. Scan fields on the thin-layer material are marked with dashed red lines; fast laser processing with AODs happens inside a single scan field, while sample movement is required to reach subsequent fields. $B E$ beam expander 
The desired microstructure shape was rasterized on rectangular grid with a variable spacing to ensure an optimal overlapping of the burst positions on the material (called spot spacing- $s$ ). Typical spot spacing used for line structures was 0.2 and $0.4-0.6 \mu \mathrm{m}$ for surface processing. The typical spot spacing parameters result in high processing speeds with respect to the device accuracy and beam diameter, being $17 \mathrm{~mm} / \mathrm{s}$ for contour lines and $0.03 \mathrm{~mm}^{2} / \mathrm{s}$ for surfaces. After rasterization, the calculated laser beam positions were sent to the AODs in a randomly mixed order. Such regime has proven to be efficient for material processing in our previous research [23]. Combined heat accumulation due to absorption of previous bursts and the resulting shielding [24] have been minimized as well in this way as subsequent bursts are spatially isolated. We have shown in our previous work [16] that during a burst, the accumulation of heat in the tightly localized volume around the laser spot on the material governs the increased ablation efficiency, while particle shielding reduces it.

We have used optical microscopy and atomic force microscopy for the sample analysis. The AFM has enabled us to measure the structure edge roughness with a single nanometer precision, while optical measurements give a broader view of the structures and scan field stitching. The complete removal of the target layer can be judged from changes of the optical properties, such as reflectivity or transmissivity.

\section{Manufacturing precision}

The limitations of precise single-micrometer-sized feature manufacture with a fiber laser source were considered, analyzed, and divided according to the source of origin. The laser source outputs a laser beam that has a distinct positioning stability and the operation in bursts of pulses results in a distinct burst energy stability. The positioning system defines both the positioning accuracy and geometrical effects due to the spot spacing of two neighboring pulses. Finally, the limiting effect of mechanical stability was predicted and experimentally verified.

Geometrical effects are a result of CAD algorithms used to define the designed structure on the material. The design is rasterized to points on a square grid with a defined interspot distance. Two neighboring burst locations on the material are, therefore, separated by that distance (labelled $s$ in Fig. 2a). As the effect of a single burst is, in general, circular in shape, this results in edge roughness $\delta$. The scaling of edge roughness with increasing spot spacing is presented in Fig. 2a.

The fiber laser design used was based on a gain-switched seed diode, multiple fiber amplification stages, and a final second harmonic generation stage. While the gain switch diodes exhibit high timing stability, defined by the electric circuitry used [25], the pulse-to-pulse energy stability is on the order of $\pm 1 \%$ [26]. Multiple amplification stages work as positive feedback loops and thus decrease the energy stability. A hypothesis was used that single pulse operation will be inferior to multi-pulse bursts in terms of burst energy stability. For example, ten pulses in a burst cause averaging effects resulting from consecutive pulses passing through the amplification stages at high repetition rates $(40 \mathrm{MHz})$. The resulting burst energy stability is thus expected to be significantly improved for multi-pulse burst mode operation.

Laser burst energy stability was measured using a Thorlabs PDA36A-EC amplified Si photodetector. The laser beam was focused with a $100 \mathrm{~mm}$ lens to achieve a spot size about one-third of the detector size, ensuring that the
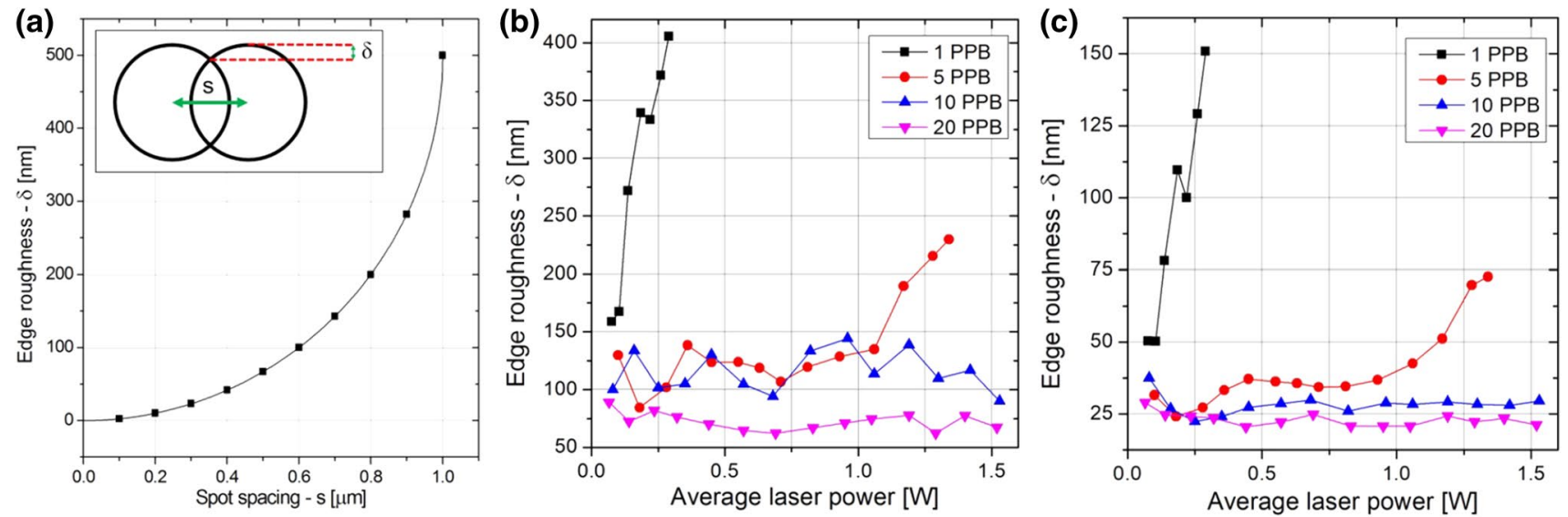

Fig. 2 a Graph of calculated structure edge roughness as a function of spot spacing for the experimentally used spot size of $1 \mu \mathrm{m}$. b Graph of structure edge roughness as a function of average laser power, calculated from the measured maximal burst-to-burst energy variations (applies to laser ablation of thin materials). c Graph of structure edge roughness as a function of average laser power, calculated from the measured standard deviation of burst energy variations (applies to laser ablation of thick materials) 
whole spatial energy distribution was inside the detector's active area. Linear response of the detector to the variations in short laser pulse energy was cross verified using a power meter to compare the measured average power with the measured average burst energy. The measured variations of the burst energy were recalculated to variations of the focused spot radius. We have used an assumption that the ablation threshold is at the steepest part of the Gaussian spatial energy distribution. The Gaussian radial energy distribution $E(r)$ was defined by the following:

$E(r)=E_{0} \mathrm{e}^{-2 r^{2} / w_{0}^{2}}$,

to calculate the radius variation $\Delta r$ caused by the burst energy variation using the following equation:

$\Delta r=\left(\frac{\partial E}{\partial r}\right)^{-1} \Delta E_{\mathrm{b}}$.

In the equations, the Gaussian beam radius is represented with $w_{0}, E_{0}$ is peak energy and $\Delta E_{\mathrm{b}}$ is burst energy variation. Combining the equations and taking into account the linear dependence of peak energy and burst energy $\left(E_{0}=2 E_{\mathrm{b}} / \mathrm{PPB}\right)$, the final radial variation of the focused Gaussian beam due to burst energy variations can be expressed as follows:

$\frac{\Delta r}{w_{0}}=\sqrt{2 \sqrt{e}} \frac{\Delta E_{\mathrm{b}}}{E_{\mathrm{b}}}$,

where $E_{\mathrm{b}}$ is the total burst energy. The variations of the effective radial size as a function of burst energy are shown in Fig. 2b, $c$ for different PPB settings. For thin materials, where a single burst removes the whole material thickness, the edge roughness is calculated from the highest measured burst-to-burst energy difference, as shown in Fig. 2b. For thick materials, where multiple bursts are needed to create the final structure edge, the edge roughness is averaged and thus calculated from the measured standard deviation of burst energy variations, as shown in Fig. 2c. Both criteria show a similar finding that operation in longer bursts of pulses ensures a more stable operation of the fiber laser source, compared at equal average powers and a fixed burst repetition rate $f_{\mathrm{b}}$. This is a direct consequence of lower peak powers reached during longer bursts and leads to a lower structure edge roughness.

The laser positioning stability was measured using a quadrant photodiode (OSI Optoelectronics, SPOT-9DMI). With the detector positioned in the focal plane of a $100 \mathrm{~mm}$ lens, we have created comparable conditions for the measurement of instability effects as after focusing through the microscope objective. The maximum measured instability was below $15 \%$ of the beam radius for all laser parameters. We have used the same laser parameter set compared to the energy stability measurements presented in Fig. $2 b$, c. The device's optical setup increases the beam radius for a factor of six before focusing, and thus, the angles are reduced equivalently. The resulting structure edge roughness due to beam pointing instability was estimated to be below $20 \mathrm{~nm}$ after focusing though a $20 \times / 0.5$ microscope objective. The highest predicted edge roughness values due to the laser source and beam guiding with AODs (taken from our previous work [16]) are summarized in Table 1. Inhomogeneity of the material itself or that of the ablation process was not taken into account.

The experimental results showing the system's precision, smallest consistently achievable feature size, and the resulting edge roughness are combined in Fig. 3. The array of holes made by a single burst per spot in a $250 \mathrm{~nm}$ layer of gold on glass stretches for several millimeters in each direction, demonstrating the capability of the system to achieve homogeneous and repeatable results over larger surfaces. The precision of AODs within a scan field is combined with a system for sample movement, sample position analysis, and precise tuning of the exact position of the next scan field with AODs. The red dashed lines mark the edges of scan field and the structure's periodicity is retained with an estimated error well below $1 \mu \mathrm{m}$. The missing holes are fabricated on purpose to stress the periodicity of the array. The line structures in an ITO layer on glass were structured to demonstrate the precise ablation of thin-layer materials, widely used in modern electronic applications (i.e., touchscreens and photovoltaics [27]). The upper part of Fig. 3b shows the effect of un-damped mechanical vibrations, leading to a worsened feature edge roughness and an increased feature size. After damping, the achieved feature size and edge roughness are in good agreement with the predicted
Table 1 Summary of measured and predicted values for structure edge roughness at different laser parameters from the identified sources

\begin{tabular}{|c|c|c|c|c|}
\hline Source of edge roughness & $1 \mathrm{PPB}$ & $5 \mathrm{PPB}$ & $10 \mathrm{PPB}$ & $20 \mathrm{PPB}$ \\
\hline Maximal burst energy variations & $\leq 400 \mathrm{~nm}$ & $\leq 200 \mathrm{~nm}$ & $\leq 140 \mathrm{~nm}$ & $\leq 80 \mathrm{~nm}$ \\
\hline $\begin{array}{l}\text { Standard deviation of the average burst } \\
\text { energy }\end{array}$ & $\leq 160 \mathrm{~nm}$ & $\leq 70 \mathrm{~nm}$ & $\leq 30 \mathrm{~nm}$ & $\leq 20 \mathrm{~nm}$ \\
\hline Laser's positioning stability & \multicolumn{4}{|l|}{$\leq 20 \mathrm{~nm}$} \\
\hline Spot spacing & \multicolumn{4}{|c|}{$10-100 \mathrm{~nm}$ (spot spacing $200-600 \mathrm{~nm}$; typical) } \\
\hline AOD beam positioning accuracy & \multicolumn{4}{|l|}{$\leq 1 \mathrm{~nm}$} \\
\hline
\end{tabular}



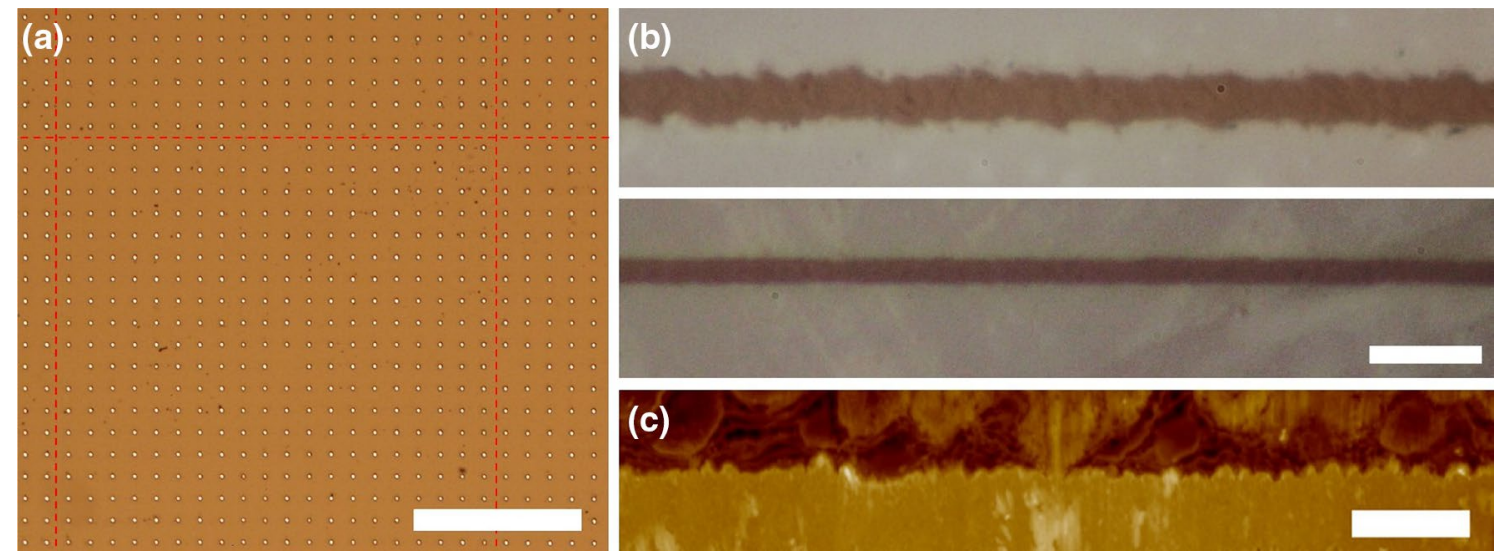

Fig. 3 a Optical image of a large array of holes, fabricated in a thin gold layer $(250 \mathrm{~nm})$ on glass using a single burst per spot. Scale bar equals to $100 \mu \mathrm{m}$. b Optical images of a laser processed ITO layer on glass, scale bar equals to $5 \mu \mathrm{m}$. Un-damped mechanical vibrations results are shown in the upper part, while vibrations were damped for the bottom part. ITO has a higher reflectivity than glass and appears

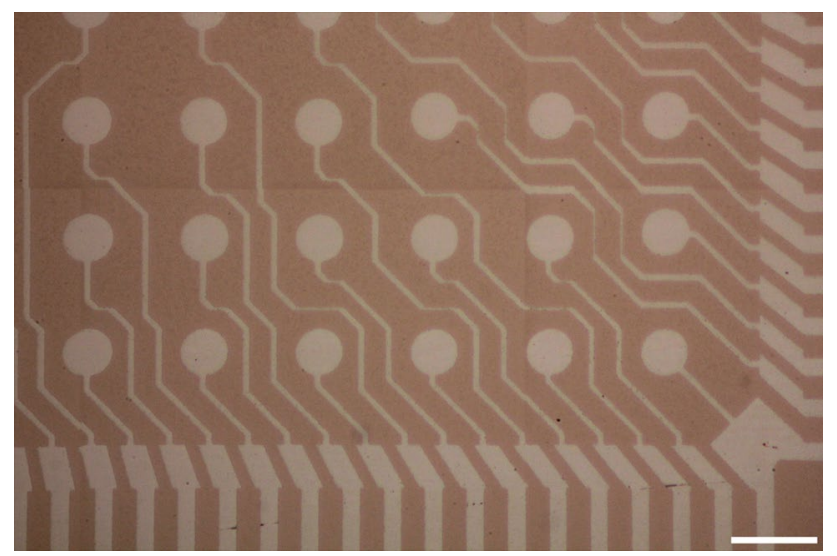

Fig. 4 Optical image of a high-precision structure manufactured in an ITO layer (brighter parts), stretching across multiple scan field seamlessly stitched together. The scale bar equals $50 \mu \mathrm{m}$

values - the spot size of $1 \mu \mathrm{m}$ leads to a typical feature size of $1 \mu \mathrm{m}$ and the edge roughness in thin material is below $200 \mathrm{~nm}$ (bottom part of Fig. 3b). AFM measurements were performed to verify the optical microscopy observations, the result is shown in Fig. 3c. Small irregular features on the structure edge (on the order of $100 \mathrm{~nm}$ ) are a result of irregularities of the material or the ablation process. The previously predicted and optically observed edge roughness of around $200 \mathrm{~nm}$ or less has been confirmed in this way, with a measured standard deviation of edge coordinate equal to $70 \mathrm{~nm}$ (using Bruker NanoScope Analysis 1.40 software and the data illustrated in Fig. 3c). The ability to create singlemicrometer-sized structures across multiple scan fields in thin-layer materials is demonstrated in Fig. 4. brighter [28]. c AFM measurement of the ITO edge roughness after laser processing, scale bar equals to $1 \mu \mathrm{m}$. Brighter parts correspond to the ITO layer. The laser was focused on the material surface in all cases, while the total fluence delivered to the material was up to $200 \mathrm{~J} / \mathrm{cm}^{2}$ due to pulse overlap

\section{Discussion and conclusions}

We have designed, evaluated, analyzed, and experimentally verified a picosecond fiber laser-based experimental device combining fast beam deflection and tight focusing. We have demonstrated that operation in bursts of 5-20 pulses improves the burst-to-burst energy stability of the laser and thus leads to an improved structure quality. This is a result of using lower pulse energies that are still sufficient for material processing applications due to both tight focusing of the laser beam and a comparable or even improved laser ablation efficiency while using bursts. The latter was demonstrated in our previous work [16]. Operation in bursts of pulses in combination with AOD-based beam steering also enables a higher throughput by increasing the effective repetition rate while working at the same repositioning frequency, which cannot be realized without burst mode operation.

We have predicted the combined effects of laser beam focusing, laser operation inaccuracy, the beam steering mechanism, and the geometrical effect of spot spacing, all together defining and limiting the typical feature size and structure edge roughness. We have confirmed the estimated values through fabrication of structures in thin-layer materials, resulting in approximately $1 \mu \mathrm{m}$-sized features with sub$200 \mathrm{~nm}$ edge roughness. The precision of scan field stitching and mechanical vibration dampening have both been verified by the experimental device setup. The flexible design of a custom fiber laser source in combination with the sophisticated beam steering setup provides a new tool for material processing and optical applications. The results show that our fiber laser setup fulfills to achieve the needed precision for processing with a single-micrometer feature size. 
Acknowledgements We acknowledge the funding received from the Slovenian Research Agency ARRS (Grants L2-6780 and L2-8183) and from the Ministry of Education, Science and Sport, Republic of Slovenia (Grant SPS GOSTOP).

\section{References}

1. B.N. Chichkov, C. Momma, S. Nolte, F. von Alvensleben, A. Tünnermann, Appl. Phys. A 63, 109 (1996)

2. M. Lenzner, J. Krüger, W. Kautek, F. Krausz, Appl. Phys. A 68, 369 (1999)

3. B. Sallé, O. Gobert, P. Meynadier, M. Perdrix, G. Petite, A. Semerok, Appl. Phys. A 69, S381 (1999)

4. F. Korte, J. Serbin, J. Koch, A. Egbert, C. Fallnich, A. Ostendorf, B.N. Chichkov, Appl. Phys. A 77, 229 (2003)

5. L. Jiang, H.-L. Tsai, J. Heat Transf 128, 926 (2006)

6. A. Ancona, S. Döring, C. Jauregui, F. Röser, J. Limpert, S. Nolte, A. Tünnermann, Opt. Lett. 34, 3304 (2009)

7. L.V. Zhigilei, Z. Lin, D.S. Ivanov, J. Phys. Chem. C 113, 11892 (2009)

8. D. Bäuerle, in Laser Processing and Chemistry (Springer, Berlin, 2011), pp. 237-278

9. K. Sugioka, Y. Cheng, Light Sci. Appl. 3, e149 (2014)

10. J. Schille, L. Schneider, U. Loeschner, Appl. Phys. A 120, 847 (2015)

11. J. Schille, L. Schneider, S. Kraft, L. Hartwig, U. Loeschner, Appl. Phys. A 122, 644 (2016)

12. C.B. Campbell, T.M. Lehecka, J.G. Thomas, V.V. Semak, in Proceedings of ICALEO 2008 (2008), pp. 181-186
13. R. Knappe, H. Haloui, A. Seifert, A. Weis, A. Nebel, in Proceedings of SPIE 7585 (2010), p. $75850 \mathrm{H}-6$

14. C. Gaudiuso, H. Kämmer, F. Dreisow, A. Ancona, A. Tünnermann, S. Nolte, in Proceedings of SPIE 9740 (2016), p. 974017-8

15. C. Kerse, H. Kalaycioğlu, P. Elahi, B. Çetin, D.K. Kesim, Ö Akçaalan, S. Yavaş, M.D. Aşık, B. Öktem, H. Hoogland, R. Holzwarth, F.Ö. Ilday, Nature 537, 84 (2016)

16. J. Mur, J. Petelin, N. Osterman, R. Petkovšek, J. Phys. Appl. Phys. 50, 325104 (2017)

17. J. Mur, L. Pirker, N. Osterman, R. Petkovšek, Opt. Express 25, 26356 (2017)

18. P. Lickschat, A. Demba, S. Weissmantel, Appl. Phys. A 123, 137 (2017)

19. N. Anscombe, Nat. Photon. 4, 22 (2010)

20. Y. Temiz, R.D. Lovchik, G.V. Kaigala, E. Delamarche, Microelectron. Eng. 132, 156 (2015)

21. C. Jauregui, J. Limpert, A. Tünnermann, Nat. Photon. 7, 861 (2013)

22. J. Petelin, B. Podobnik, R. Petkovšek, Appl. Opt 54, 4629 (2015)

23. J. Mur, B. Podobnik, I. Poberaj, Opt. Laser Technol. 88, 140 (2017)

24. N. Bellini, R. Geremia, D. Karnakis, Appl. Phys. A 123, 346 (2017)

25. A.J. Taylor, J.M. Wiesenfeld, G. Eisenstein, R.S. Tucker, Appl. Phys. Lett. 49, 681 (1986)

26. R. Petkovšek, V. Agrež, Opt. Express 22, 1366 (2014)

27. K. Ellmer, Nat. Photon. 6, 809 (2012)

28. R.J. Moerland, J.P. Hoogenboom, Optica 3, 112 (2016) 